\title{
SPLITTING AND MELNIKOV POTENTIALS IN HAMILTONIAN SYSTEMS
}

\author{
AMADEU DELSHAMS AND PERE GUTIÉRREZ \\ Departament de Matemàtica Aplicada I, Universitat Politècnica de Catalunya, \\ Diagonal 647, 08028 Barcelona
}

\begin{abstract}
We consider a perturbation of an integrable Hamiltonian system, possessing hyperbolic invariant tori with coincident whiskers. Following an idea due to Eliasson, we introduce a splitting potential whose gradient gives the splitting distance between the perturbed stable and unstable whiskers. The homoclinic orbits to the perturbed whiskered tori are the critical points of the splitting potential, and therefore their existence is ensured in both the regular (or strongly hyperbolic, or a-priori unstable) and the singular (or weakly hyperbolic, or a-priori stable) case. The singular case is a model of a nearly-integrable Hamiltonian near a single resonance. In the regular case, the Melnikov potential is a first order approximation of the splitting potential, and the standard Melnikov (vector) function is simply the gradient of the Melnikov potential. Non-degenerate critical points of the Melnikov potential give rise to transverse homoclinic orbits. Explicit computations are carried out for some examples.
\end{abstract}

\section{Introduction}

For more than 2 degrees of freedom, the problem of measuring the splitting of the whiskers of hyperbolic invariant tori is closely related with the existence of instability in nearly-integrable Hamiltonian systems, i.e. with the Arnold diffusion. In this lecture, the splitting is studied in a wide setting, and a general Poincaré-Melnikov theory is developed.

Setup

We start with a perturbation of a hyperbolic integrable Hamiltonian, with $n+1 \geq 3$ degrees of freedom. In canonical variables $z=(x, y, \varphi, I) \in D \subset$ $\mathbb{T} \times \mathbb{R} \times \mathbb{T}^{n} \times \mathbb{R}^{n}$, with the symplectic form $\mathrm{d} x \wedge \mathrm{d} y+\mathrm{d} \varphi \wedge \mathrm{d} I$, consider a Hamiltonian of the form

$$
\begin{aligned}
& H(x, y, \varphi, I ; \mu)=H_{0}(x, y, I)+\mu H_{1}(x, y, \varphi, I) \\
& H_{0}(x, y, I)=\langle\omega, I\rangle+\frac{1}{2}\langle\Lambda I, I\rangle+\frac{y^{2}}{2}+V(x)+\langle\lambda, I\rangle y
\end{aligned}
$$


where $\mu$ is a perturbation parameter. The Hamiltonian equations associated to $H$ are:

$$
\begin{array}{ll}
\dot{x}=y+\langle\lambda, I\rangle & +\mu \partial_{y} H_{1}(x, y, \varphi, I), \\
\dot{y}=-V^{\prime}(x) & -\mu \partial_{x} H_{1}(x, y, \varphi, I), \\
\dot{\varphi}=\omega+\Lambda I+\lambda y & +\mu \partial_{I} H_{1}(x, y, \varphi, I), \\
\dot{I}= & -\mu \partial_{\varphi} H_{1}(x, y, \varphi, I) .
\end{array}
$$

It will shown in section 2 that, under weak assumptions, the unperturbed Hamiltonian $H_{0}$ has $n$-dimensional whiskered tori (hyperbolic invariant tori) with coincident $(n+1)$-dimensional whiskers (invariant manifolds). For a given whiskered torus of $H_{0}$, its (unique) whisker is filled by homoclinic orbits (biasymptotic to the torus). Our aim is to study the splitting of the whiskers, and the persistence of some homoclinic orbits, for $\mu \neq 0$.

\section{Main achievements}

To deal with this problem, the tools used are Poincaré-Melnikov theory, and a geometric method based on Eliasson's approach. Our contributions can be summarized as follows:

- A general Poincaré-Melnikov theory for Hamiltonian systems is developed, defining a scalar function $L$ (Melnikov potential) whose gradient $M$ (Melnikov function) gives the splitting distance at first order in $\mu$.

- There exists a scalar function $\mathcal{L}$ (splitting potential) such that, in suitable variables, its gradient $\mathcal{M}$ (splitting function) gives exactly the splitting distance. Besides, the splitting potential $\mathcal{L}$ is approximated at first order in $\mu$ by the Melnikov potential $L$.

- The results are significant for more than 2 degrees of freedom.

\section{Motivation}

The study of the splitting in the Hamiltonian (1-2) is closely related to the problem of Arnold diffusion in a general nearly-integrable Hamiltonian system:

$$
H(\phi, J)=h(J)+\varepsilon f(\phi, J),
$$

in angle-action variables $(\phi, J) \in \mathbb{T}^{n+1} \times \mathbb{R}^{n+1}$. Here, the small perturbation parameter is $\varepsilon$.

Near single resonances, it is known ${ }^{1,2,3}$ that one step of (resonant) normal form procedure can be performed and leads, under some generic hypotheses 
and after a scaling, to a Hamiltonian of the type (1-2), taking as $H_{0}$ the truncated normal form.

To make this clearer, consider a selected action $J^{*}=0$, and assume that its associated frequency vector $\partial_{J} h(0) \in \mathbb{R}^{n+1}$ has a single resonance (this means $\left\langle k^{*}, \partial_{J} h(0)\right\rangle=0$ for a certain $k^{*} \in \mathbb{Z}^{n+1} \backslash\{0\}$ and $\left\langle k, \partial_{J} h(0)\right\rangle \neq 0$ for any $k \in \mathbb{Z}^{n+1}$ not co-linear to $\left.k^{*}\right)$. It can be assumed that $\partial_{J} h(0)=\left(0, \omega^{*}\right)$, with $\omega^{*} \in \mathbb{R}^{n}$ nonresonant. Near $J^{*}$, the unperturbed Hamiltonian $h$ in (3) can be written as:

$$
h(J)=\left\langle\partial_{J} h(0), J\right\rangle+\frac{1}{2}\left\langle\partial_{J}^{2} h(0) J, J\right\rangle+\mathrm{O}_{3}(J) .
$$

We write $\phi=(x, \varphi) \in \mathbb{T} \times \mathbb{T}^{n}$ and $J=(y, I) \in \mathbb{R} \times \mathbb{R}^{n}$, and

$$
\partial_{J}^{2} h(0)=\left(\begin{array}{cc}
\beta^{2} & \lambda^{\top} \\
\lambda & \Lambda
\end{array}\right)
$$

where we have put $\beta^{2}>0$ in order to fix ideas, $\lambda \in \mathbb{R}^{n}$, and $\Lambda$ is an $(n \times n)$ matrix. With some scaling, we can assume $\beta=1$, and our Hamiltonian written in the form

$$
\begin{aligned}
& H(x, y, \varphi, I)=h(y, I)+\varepsilon f(x, y, \varphi, I), \\
& h(y, I)=\left\langle\omega^{*}, I\right\rangle+\frac{1}{2}\langle\Lambda I, I\rangle+\frac{y^{2}}{2}+\langle\lambda, I\rangle y+\mathrm{O}_{3}(y, I) .
\end{aligned}
$$

Performing one step of resonant normal form procedure, we can construct a symplectic map $\Phi$ such that $H \circ \Phi=H_{0}+H_{1}$, with

$$
\begin{aligned}
& H_{0}(x, y, I ; \varepsilon)=\left\langle\omega^{*}, I\right\rangle+\frac{1}{2}\langle\Lambda I, I\rangle+\frac{y^{2}}{2}+\varepsilon V(x)+\langle\lambda, I\rangle y, \\
& H_{1}(x, y, \varphi, I ; \varepsilon)=\varepsilon R(x, y, \varphi, I)+\mathrm{O}_{3}(y, I)+\mathrm{O}\left(\varepsilon^{2}\right),
\end{aligned}
$$

and $V(x)$ is the periodic function obtained by averaging with respect to the angles $\varphi$ :

$$
V(x)=\bar{f}(x, 0,0)=\frac{1}{(2 \pi)^{n}} \int_{\mathbb{T}^{n}} f(x, 0, \varphi, 0) \mathrm{d} \varphi, \quad x \in \mathbb{T} .
$$

In the normalized expression for $H$, note that $H_{0}$ (the truncated normal form) is an integrable Hamiltonian, and then $H_{1}$ can be considered as a perturbation of some size $\varepsilon \mu$ where $\mu$ can be determined in terms of $\varepsilon$. In this sense, the expression obtained generalizes the Lochak's example ${ }^{4}$ (which, in its turn, generalizes the famous Arnold's example, ${ }^{5}$ designed to describe the diffusion).

Under generic hypotheses, it can be shown that the Hamiltonian $H_{0}$ has whiskered tori with coincident whiskers associated to this hyperbolic point (see 
section 2). Therefore, although there is no hyperbolicity in $h$, the perturbation $f$ provides some weak hyperbolicity, which appears in the truncated normal form $H_{0}$. This hyperbolicity disappears for $\varepsilon \rightarrow 0$, because the Lyapunov exponents of the whiskered tori of $H_{0}$ are of the form $\pm \sqrt{\varepsilon} \alpha$. To have fixed exponents, we replace $y, I$ by $\sqrt{\varepsilon} y, \sqrt{\varepsilon} I$ (a non-canonical linear change), and divide the Hamiltonian by $\varepsilon$. Then the new system is still Hamiltonian, and we obtain obtain for $H=H_{0}+H_{1}$ an expression of the form (1-2), with

$$
\omega=\frac{\omega^{*}}{\sqrt{\varepsilon}}, \quad \mu=\mathrm{O}\left(\varepsilon^{1 / 2}\right) .
$$

It has to be pointed out that, after this procedure, in general the truncated normal form $H_{0}$ is a coupled Hamiltonian: $\lambda \neq 0$ in (2). So the motivation for the coupling term $\langle\lambda, I\rangle y$ is that this term appears in a natural way when one studies a nearly-integrable Hamiltonian, in a region close to a single resonance. As a particular case, note that if $\lambda=0$ in (2), then the unperturbed Hamiltonian $H_{0}$ is somewhat simpler because it is formed by a pendulum and $n$ rotors: we then say that $H_{0}$ is uncoupled. We will show in section 4 that the formulation of Poincaré-Melnikov theory is simpler in this special case.

Although the (homoclinic) splitting between the whiskers of hyperbolic tori in single resonances is very important in the detection of Arnold diffusion (through the construction of transition chains), we point out that there are other important difficulties related with this problem. These difficulties are the study of the transition properties of the tori, the detection of heteroclinic intersections between whiskers of different tori, and jumping the gaps associated to double resonances.

Regular and singular cases

According to the motivation above, it is convenient in (1-2) to allow $\omega$ to depend on an additional parameter $\varepsilon$, considering fast frequencies $\omega=\omega^{*} / \sqrt{\varepsilon}$. The parameters $\varepsilon$ and $\mu$ can be whether independent or linked by a relation of the type $\mu=\varepsilon^{p}$ with some $p>0$; these two cases will be called, respectively, regular and singular. We have shown that, in the study of a general nearlyintegrable Hamiltonian, the actually relevant case is the singular one (with $p=1 / 2$ ), and that this feature is directly related to the weak hyperbolicity of the truncated normal form.

Concerning the regular situation, we recall that the strategy of keeping $\varepsilon>0$ fixed and letting $\mu \rightarrow 0$ (having in this way a regular system) was introduced by Arnold ${ }^{5}$ in order to avoid dealing with a singular perturbation problem. In this case, Poincaré-Melnikov theory can be applied directly to the 
detection of the splitting, but only if the parameter $\mu$ is taken exponentially small with respect to $\varepsilon$. This is due to that the Melnikov integrals involved are exponentially small in $\varepsilon$, as in the second example shown in section 5 (for the first example shown, the integrals are not exponentially small, because the perturbation is not analytic in this case).

In the singular case, one assumes that the parameters $\varepsilon$ and $\mu$ satisfy a power-like relation of the type $\mu=\varepsilon^{p}$ (the smaller $p$ the better), and one lets $\varepsilon \rightarrow 0$. In this case, the problem of detecting the splitting from the Melnikov integrals is much more intricate, because of the exponentially small character of the integrals involved. However, some recent works ${ }^{6,7,8}$ suggest that, under some weak conditions, the Melnikov integrals give the right predictions for the splitting.

Nevertheless, the existence of homoclinic orbits has been established in several works. ${ }^{1,9,10}$ This result is valid for regular and singular systems, and we recall it in section 6 .

\section{The unperturbed Hamiltonian}

\section{Assumptions}

In this section, we take $\mu=0$ and study the unperturbed Hamiltonian $H_{0}$ defined in (2). Note that the given ingredients of $H_{0}$ are the vectors $\omega, \lambda \in \mathbb{R}^{n}$, the symmetric $(n \times n)$-matrix $\Lambda$, and the function $V(x)$ of $x \in \mathbb{T}$. We require the following assumptions:

- The function $V(x)$ has a unique and nondegenerate global maximum. To fix ideas, we require

$$
\begin{aligned}
& V(0)=0, \quad V^{\prime}(0)=0, \quad V^{\prime \prime}(0)<0 \\
& V(x)<0 \quad \forall x \neq 0 \quad(\bmod 2 \pi) .
\end{aligned}
$$

- The following nondegeneracy condition holds:

$$
\operatorname{det}\left(\Lambda-\lambda \lambda^{\top}\right)=\operatorname{det}\left(\begin{array}{cc}
1 & \lambda^{\top} \\
\lambda & \Lambda
\end{array}\right) \neq 0 .
$$

- The vector $\omega$ is assumed to satisfy a Diophantine condition: for some $\tau \geq n-1$ and $\gamma>0$,

$$
|\langle k, \omega\rangle| \geq \gamma|k|^{-\tau} \quad \forall k \in \mathbb{Z}^{n} \backslash\{0\} .
$$


The unperturbed torus and its homoclinic whisker

The integrable Hamiltonian $H_{0}$ can easily be studied. Let us introduce

$$
P(x, y)=\frac{y^{2}}{2}+V(x), \quad \widehat{P}(x, y, I)=P(x, y+\langle\lambda, I\rangle) ;
$$

then $H_{0}$ can be rewritten as

$$
H_{0}=\langle\omega, I\rangle+\frac{1}{2}\left\langle\left(\Lambda-\lambda \lambda^{\top}\right) I, I\right\rangle+\widehat{P}(x, y, I) .
$$

We see that, on every plane $I=$ const, the Hamiltonian $H_{0}$ reduces to a 1 degree-of-freedom Hamiltonian: a generalized pendulum (the standard pendulum being given by $V(x)=\cos x-1)$. This pendulum has $(x, y)=(0,-\langle\lambda, I\rangle)$ as a hyperbolic equilibrium point, with (homoclinic) separatrices given by $y+\langle\lambda, I\rangle= \pm \sqrt{-2 V(x)}$. The Lyapunov exponents of the hyperbolic point are $\pm \alpha$, where we define $\alpha=\sqrt{-V^{\prime \prime}(0)}$.

Therefore, the Hamiltonian $H_{0}$ possesses an $n$-parameter family of $n$ dimensional whiskered tori given by the equations $I=$ const, $y=-\langle\lambda, I\rangle, x=$ 0 , with $(n+1)$-dimensional whiskers. The stable and unstable whiskers of each torus coincide, and hence all orbits on this (unique) whisker are homoclinic, i.e. biasymptotic to the torus.

We will focus our attention on a concrete hyperbolic torus, that we assume located at the origin: $I=0, x=y=0$. Note that the vector $\omega$, assumed Diophantine, consists of the frequencies of this torus: $\dot{\varphi}=\omega$. In view of the nondegeneracy condition (5), the neighbor tori have different frequencies.

\section{Parameterizations for the unperturbed Hamiltonian}

We denote $\mathcal{T}_{0}$ the whiskered torus of $H_{0}$ having frequency vector $\omega$. This torus can obviously be parameterized by

$$
\mathcal{T}_{0}: \quad z_{0}^{*}(\varphi)=(0,0, \varphi, 0), \quad \varphi \in \mathbb{T}^{n} .
$$

As mentioned above, the stable and unstable whiskers of the torus $\mathcal{T}_{0}$ coincide; this homoclinic whisker is given by the equations $I=0, P(x, y)=0$. We denote $\mathcal{W}_{0}$ the positive part $(y>0)$ of the homoclinic whisker (it is often called separatrix). To give a suitable parameterization for $\mathcal{W}_{0}$, we consider the 1-degree-of-freedom Hamiltonian $P(x, y)$, and denote $\left(x_{0}(s), y_{0}(s)\right)$ the associated homoclinic trajectory, with $x_{0}(0)=\pi, y_{0}(0)>0$. Note that $x_{0}(s)$ goes from 0 to $2 \pi$ when $s$ goes from $-\infty$ to $\infty$. It is clear that we can give the whisker $\mathcal{W}_{0}$ the parameterization

$$
\mathcal{W}_{0}: \quad z_{0}(s, \varphi)=\left(x_{0}(s), y_{0}(s), \varphi+\left(x_{0}(s)-\pi\right) \lambda, 0\right), \quad s \in \mathbb{R}, \quad \varphi \in \mathbb{T}^{n}
$$


where the term $\left(x_{0}(s)-\pi\right) \lambda$ expresses the phase drift undergone by any trajectory when traveling along $\mathcal{W}_{0}$. This drift is associated to the coupling term. Note that, with our definition, the dynamics on $\mathcal{W}_{0}$ is given by the equations $\dot{s}=1, \dot{\varphi}=\omega$. One has

$$
\lim _{t \rightarrow \pm \infty}\left[z_{0}(s+t, \varphi+\omega t)-z_{0}^{*}(\varphi \pm \pi \lambda+\omega t)\right]=0,
$$

and this implies that that every trajectory on $\mathcal{W}_{0}$ is biasymptotic to two different trajectories on the invariant torus $\mathcal{T}_{0}$. If $\lambda$ is an integer (a very special case) then these two trajectories on $\mathcal{T}_{0}$ coincide.

\section{Preservation of the whiskered torus and its whiskers}

The local normal form

Before studying the splitting, we have to establish the surviving under perturbations of our Diophantine whiskered torus, as well as its local whiskers. Then we have to extend them to global whiskers in order to compare the stable and the unstable ones. The surviving of the torus and its local whiskers under a small perturbation can be ensured by means of the hyperbolic KAM theorem, a version of the KAM theorem adapted to this problem.

Roughly speaking, the hyperbolic KAM theorem provides a symplectic transformation $\Phi$ taking our Hamiltonian into a local normal form $\widetilde{H}=H \circ \Phi$ (in some domain), having a simpler expression in which the perturbed torus becomes transparent, as well as its whiskers. This kind of result follows from a convergent KAM-like iterative scheme.

We are interested in a normal form defined in a whole neighborhood of our concrete torus, ${ }^{1,11}$ according to the "Kolmogorov's approach" to KAM theory. This approach allows us to control a neighborhood of the local stable whisker, which can be ensured in this way to contain also a piece of the global stable whisker (this feature is used in section 6). On the contrary, in the "Arnold's approach" (used in other papers) the normal form only holds on a Cantor set, although a large family of surviving tori is obtained. Some more comments and references to papers following both approaches are given in a recent paper of the authors. ${ }^{10}$

In most papers (like for instance ${ }^{11}$ ), the hyperbolic KAM theorem is dealt in terms of some local variables in a neighborhood of the torus, in such a way that the whiskers become coordinate planes. A significantly new approach was introduced by Eliasson, who rewrote the hyperbolic KAM theorem and expressed it directly in the "original variables". 1 This is more suitable to our 
purpose of carrying out a global control of the whiskers in order to study their splitting (see section 6).

Another key fact is the use of exact symplectic transformations to normal form in the hyperbolic KAM theorem. To recall what an exact symplectic transformation is, consider the 1 -form $\eta=-(y \mathrm{~d} x+I \mathrm{~d} \varphi)$, whose differential is the standard symplectic 2-form: $\mathrm{d} \eta=\mathrm{d} x \wedge \mathrm{d} y+\mathrm{d} \varphi \wedge \mathrm{d} I$. Then a transformation $\Phi$ is symplectic if the 1-form $\Phi^{*} \eta-\eta$ is closed, and it is exact symplectic if this 1 -form is exact $(=\mathrm{d} S$, globally, for some scalar primitive $S)$.

Eliasson ${ }^{1}$ used the exactness of the normalizing transformation as a crucial tool in order to detect homoclinic intersections between the whiskers, in both regular and singular systems (although he did not compute the splitting). A similar result was also obtained by Bolotin. ${ }^{9}$ In a further step, in the present lecture the exactness allows us to put the splitting function as the gradient of a splitting potential (see section 6).

Another paper that has influenced our version of Eliasson's theorem is a recent one by Niederman. ${ }^{11}$ This paper deals with a similar framework (using the Kolmogorov's approach but not working in the original variables), and obtains more accurate estimates for the normal form.

Let us introduce first some notations. Concerning the domain, we define for $r>0$ the complex set

$$
B_{r}=\{(x, y, \varphi, I):|x|,|y|,|I|,|\operatorname{Im} \varphi| \leq r\} .
$$

For a function $f(x, y, \varphi, I)$ analytic on some domain $D$ (and continuous on its closure), we denote $|f|_{D}$ its supremum norm.

Theorem 1 (Eliasson's theorem) Let $H=H_{0}+\mu H_{1}$ as described in (12) and in the assumptions (4-6), with $\tau>n-1$. Assume $H$ analytic on $B_{r}\left(r \leq r_{0}\right)$. Then for $|\mu|$ small enough, there exists an exact symplectic transformation $\Phi=\Phi(\cdot ; \mu): B_{\nu r} \longrightarrow B_{r}$ (analytic with respect to $(x, y, \varphi, I)$ and $\mu$ ), $0<\nu<1$, and there exist $a=a(\mu), b=b(\mu)$ (analytic in $\mu$ ), such that $\widetilde{H}=H \circ \Phi$ takes the form

$$
\widetilde{H}=\langle\omega, I-a\rangle+b \widehat{P}(x, y, I)+\mathrm{O}_{2}(\widehat{P}(x, y, I), I-a) .
$$

Besides, one has $\Phi=\mathrm{id}+\mathrm{O}(\mu), a=\mathrm{O}(\mu), b=1+\mathrm{O}(\mu)$.

The most important point about this result is that, thanks to the use of the original variables $x, y$, the local normal form $\widetilde{H}$ can be put in terms of the generalized pendulum $\widehat{P}(x, y, I)$. By using this feature, a "global" control of the whiskers, very useful in order to compare them and study the splitting, can be carried out. 1,10

In is not hard ${ }^{10}$ to establish the validity of theorem 1 in the singular case, with $\mu=\varepsilon^{p}$ and $\omega=\omega^{*} / \sqrt{\varepsilon}$, for $|\varepsilon|$ small enough. 
Parameterization of the perturbed torus

It is clear that the normal form $\widetilde{H}$ given in (8) has a whiskered torus of frequency vector $\omega$. We denote this torus as $\widetilde{\mathcal{T}}$, and its associated local whiskers as $\widetilde{\mathcal{W}}_{\text {loc }}^{+}$(stable) and $\widetilde{\mathcal{W}}_{\text {loc }}^{-}$(unstable).

The torus $\widetilde{\mathcal{T}}$ has the following obvious parameterization:

$$
\widetilde{\mathcal{T}}: \quad \tilde{z}^{*}(\varphi)=(0,-\langle\lambda, a\rangle, \varphi, a), \quad \varphi \in \mathbb{T}^{n} .
$$

This torus can be translated to a whiskered torus $\mathcal{T}$ of the original perturbed Hamiltonian $H$ :

$$
\mathcal{T}: \quad z^{*}(\varphi)=\Phi\left(\tilde{z}^{*}(\varphi)\right), \quad \varphi \in \mathbb{T}^{n} .
$$

In section 4 , it will be useful to give a first order approximation in $\mu$ for the shift suffered by the perturbed torus $\mathcal{T}$ with respect to the unperturbed torus $\mathcal{T}_{0}$, along the $I$-direction. We will denote $I^{*}(\varphi)$ the $I$-component of $z^{*}(\varphi)$. To describe this approximation, we consider the (zero average) scalar function $\chi(\varphi)$ solving the following small divisors equation:

$$
\left\langle\omega, \partial_{\varphi} \chi\right\rangle+\overline{H_{1}}(0,0,0)=H_{1}(0,0, \cdot, 0) .
$$

where the notation $\bar{f}$ denotes the $\varphi$-average of a function $f$. The existence of $\chi$ is ensured by the Diophantine condition (6).

The function $\chi$, introduced by Treschev, ${ }^{12}$ provides a first order approximation ${ }^{10}$ for the perturbed torus:

$$
I^{*}(\varphi)=\mu\left(\zeta-\partial_{\varphi} \chi(\varphi)\right)+\mathrm{O}\left(\mu^{2}\right), \quad \varphi \in \mathbb{T}^{n},
$$

where we define $\zeta=-\left(\Lambda-\lambda \lambda^{\top}\right)^{-1}\left(\overline{\partial_{I} H_{1}-\lambda \partial_{y} H_{1}}\right)(0,0,0)$.

Parameterizations of the perturbed whiskers

As in section 2, we can also take parameters on the perturbed local whiskers of the normal form $\widetilde{H}$ :

$$
\widetilde{\mathcal{W}}_{\mathrm{loc}}^{ \pm}: \quad \tilde{z}(s, \varphi)=\left(x_{0}(b s), y_{0}(b s)-\langle\lambda, a\rangle, \varphi+\left(x_{0}(b s)-\pi\right) \lambda, a\right),
$$

for $\pm s \geq s_{0}, \varphi \in \mathbb{T}^{n}$, with some $s_{0}=s_{0}(r)$. For the original Hamiltonian $H$, the local whiskers can be parameterized as follows:

$$
\mathcal{W}_{\mathrm{loc}}^{ \pm}: \quad z_{\mathrm{loc}}^{ \pm}(s, \varphi)=\Phi(\tilde{z}(s, \varphi)), \quad \pm s \geq s_{0}, \quad \varphi \in \mathbb{T}^{n} .
$$

In the parameters $s, \varphi$, the dynamics of $H$ on $\mathcal{W}_{\text {loc }}^{ \pm}$is given by $\dot{s}=1, \dot{\varphi}=\omega$.

We need to extend these local whiskers to global whiskers, in order to measure the splitting between them. The parameterizations of the whiskers 
$\mathcal{W}_{\text {loc }}^{ \pm}$, valid for $\pm s \geq s_{0}$, can easily be extended to further values of $s$ in a natural way, since the whiskers are formed by trajectories associated to our Hamiltonian $H$. We denote $\mathcal{W}^{ \pm}$the extended or global whiskers. These global whiskers remain at distance $\mathrm{O}(\mu)$ to the unperturbed whisker $\mathcal{W}_{0}$, for an interval of real values of the parameter $s$. This interval can be chosen large enough in order to make it possible to compare the whiskers $\mathcal{W}^{+}$and $\mathcal{W}^{-}$far from the torus $\mathcal{T}$.

\section{Poincaré-Melnikov theory}

In this section, we develop Poincaré-Melnikov theory in order to give a first order approximation for the splitting of the separatrix $\mathcal{W}_{0}$ into the perturbed whiskers $\mathcal{W}^{ \pm}$associated to the perturbed torus $\mathcal{T}$. Besides, we want to describe the set $\mathcal{W}^{+} \cap \mathcal{W}^{+}$, i.e. the homoclinic orbits to $\mathcal{T}$.

\section{Melnikov potential and Melnikov function}

In order to provide a first order approximation for the splitting, we introduce the (scalar) Melnikov potential $L(\varphi)$ and its gradient, the Melnikov function $M(\varphi)=\partial_{\varphi} L(\varphi)$, by means of improper integrals, $\mu$-independent and periodic in $\varphi \in \mathbb{T}^{n}$. These integrals are always absolutely convergent, thanks to the fact that the phase drift along the separatrix (due to the coupling term in (2)) and the first order deformation of the perturbed hyperbolic tori are taken into account.

We stress that our use of absolutely convergent integrals in the formulation of the general Poincaré-Melnikov theory for whiskered tori makes a difference with respect to some previous works, ${ }^{13,14,15}$ where conditionally convergent integrals are used and the integration limits have to be carefully chosen.

Next we define these functions in several cases, in increasing order of complexity.

- The simplest case is that of a perturbation vanishing on the whiskered torus, $H_{1}=\mathrm{O}_{2}(x, y, I)$. In this case, the whiskered torus remains unchanged $\left(\mathcal{T}=\mathcal{T}_{0}\right)$. We define the Melnikov potential through the following integral:

$$
\begin{aligned}
L(\varphi) & =-\int_{-\infty}^{\infty}\left(H_{1}-\overline{H_{1}}\right)\left(z_{0}(t, \varphi+\omega t)\right) \mathrm{d} t \\
& =-\int_{-\infty}^{\infty} H_{1}\left(z_{0}(t, \varphi+\omega t)\right) \mathrm{d} t+\text { const. }
\end{aligned}
$$


Note that the additive constant is such that $\bar{L}=0$. For the Melnikov function, it is clear that

$$
M(\varphi)=\partial_{\varphi} L(\varphi)=-\int_{-\infty}^{\infty} \partial_{\varphi} H_{1}\left(z_{0}(t, \varphi+\omega t)\right) \mathrm{d} t .
$$

The absolute convergence of the integral (12) for the Melnikov function was already pointed out by Robinson, ${ }^{15}$ stressing that in other cases this integral is only conditionally convergent. We also recall that, for $n=1$, the Melnikov potential (11) coincides with a formula given by Delshams and Ramírez-Ros. ${ }^{16}$

- Now, we consider the uncoupled case $\lambda=0$ (note that this case intersects but does not include the previous one). In this case, we define the Melnikov potential through the following integral, also absolutely convergent:

$$
\begin{aligned}
L(\varphi) & =-\int_{-\infty}^{\infty}\left[\left(H_{1}-\overline{H_{1}}\right)\left(z_{0}(t, \varphi+\omega t)\right)-\left(H_{1}-\overline{H_{1}}\right)\left(z_{0}^{*}(\varphi+\omega t)\right)\right] \mathrm{d} t \\
& =-\int_{-\infty}^{\infty}\left[H_{1}\left(z_{0}(t, \varphi+\omega t)\right)-H_{1}\left(z_{0}^{*}(\varphi+\omega t)\right)\right] \mathrm{d} t+\text { const } .
\end{aligned}
$$

- Finally, in the general case (which includes the two previous ones), we define

$$
L(\varphi)=-\int_{-\infty}^{\infty}\left(H_{1}-\overline{H_{1}}-\left\{\chi, H_{0}\right\}\right)\left(z_{0}(t, \varphi+\omega t)\right) \mathrm{d} t .
$$

Recall that $\overline{H_{1}}(x, y, I)$ denotes the $\varphi$-average of $H_{1}$, and that the function $\chi(x, y, \varphi, I)=\chi(\varphi)$ is the (zero average) function solving the small divisors equation (9). Notice that $\bar{L}=0$, because the function inside the integral has zero average. The absolute convergence of the Melnikov integral (13) can be ensured using that the function $H_{1}-\overline{H_{1}}-\left\{\chi, H_{0}\right\}$ vanishes on $\mathcal{T}_{0}$, together with the fact that $\mathcal{W}_{0}$ tends to $\mathcal{T}_{0}$ with exponentially decreasing bounds.

We remark that the formula (13) is useful in both the coupled and the uncoupled cases (in (2), $\lambda \neq 0$ and $\lambda=0$ respectively). An example illustrating the uncoupled case was given by the authors. ${ }^{10}$ Related expressions, also valid in both cases, were previously obtained by Treschev. ${ }^{12}$ In that paper, although the Melnikov potential was not introduced, the Melnikov function was expressed with the help of some correcting terms giving rise to the absolute convergence. We have improved that expression, including the correcting terms in the integral and providing a more compact formula. 
First order approximation for the splitting distance

The following standard result ${ }^{10}$ shows that a first order approximation for the splitting between the global whiskers $\mathcal{W}^{ \pm}$, measured along the $I$-direction, is given in terms of the Melnikov function $M=\partial_{\varphi} L$. Since both whiskers are $(n+1)$-dimensional manifolds contained in the same $(2 n+1)$-dimensional level of energy, it is enough to express its distance by an $n$-dimensional measure. We take the difference $I^{-}-I^{+}$as the measure for the splitting (we denote $I^{ \pm}(s, \varphi)$ the $I$-component of the parameterizations $\left.z^{ \pm}(s, \varphi)\right)$.

Theorem 2 Assuming $|\boldsymbol{\mu}|$ small enough, one has for any $|s| \leq s_{0}$ and $\varphi \in \mathbb{T}^{n}$ the following approximation:

$$
I^{-}(s, \varphi)-I^{+}(s, \varphi)=\mu \partial_{\varphi} L(\varphi-\omega s)+\mathrm{O}\left(\mu^{2}\right) .
$$

We stress that the Hamiltonian character of the equations implies the fact that the first order approximation of the splitting is simply the gradient of a scalar function $L$.

An important fact in this theorem is that $I_{1}, \ldots, I_{n}$ are first integrals of $H_{0}$. Following Treschev, ${ }^{12}$ it is not difficult to generalize theorem 2 by considering any given first integral $F$ of the unperturbed Hamiltonian $H_{0}$. Thus, it can be given an analogous first order approximation for $F^{-}(s, \varphi)-$ $F^{+}(s, \varphi)$, i.e. for the difference of the values of $F$ at the points $z^{ \pm}(s, \varphi)$. In the particular case $F=\widehat{P}$ (the function defined in (7)), one gets

$\widehat{P}^{-}(s, \varphi)-\widehat{P}^{+}(s, \varphi)=-\mu\left\langle\omega, \partial_{\varphi} L(\varphi-\omega s)\right\rangle+\mathrm{O}\left(\mu^{2}\right)=\mu \partial_{s} \tilde{L}(s, \varphi)+\mathrm{O}\left(\mu^{2}\right)$,

where we define $\tilde{L}(s, \varphi)=L(\varphi-\omega s)$, which can be considered a function defined on the separatrix $\mathcal{W}_{0}$. Using this fact, an alternative measure for the splitting could be $I_{1}^{-}-I_{1}^{+}, \ldots, I_{n-1}^{-}-I_{n-1}^{+}, \widehat{P}^{-}-\widehat{P}^{+}\left(\right.$if $\left.\omega_{n} \neq 0\right)$, instead of $I_{1}^{-}-I_{1}^{+}, \ldots, I_{n}^{-}-I_{n}^{+}$.

\section{Transverse homoclinic orbits}

As a simple corollary of theorem 2, we see that in the regular case the simple zeros of the Melnikov function $M$ give rise, for $|\mu|$ small enough, to transverse homoclinic intersections between the perturbed whiskers. As is well-known, if a point belongs to the homoclinic intersection, then its whole orbit is also contained in this intersection. Thus, it is enough to find the zeros of $M(\varphi-\omega s)$ for a fixed value of $s$ (a $\varphi$-section), and from the simple zeros of $M$ we get transverse homoclinic orbits biasymptotic to the perturbed torus (contained in both the stable and the unstable whiskers).

Since $M$ is the gradient of the Melnikov potential $L$, it is obvious that the simple zeros of $M$ are the nondegenerate critical points of $L$. If the function $L$ 
(defined on $\mathbb{T}^{n}$ ) is a Morse function (its critical points are all nondegenerate: a generic property), we deduce from Morse theory that for $|\mu|$ small enough there exist at least $2^{n}$ transverse homoclinic orbits.

It is well-known that this argument does not apply in the singular case, $\omega=\omega^{*} / \sqrt{\varepsilon}$ and $\mu=\varepsilon^{p}$, because the Melnikov function $M$ is typically exponentially small in $\varepsilon$ (see the second example in section 5 ). To ensure that $\mu M(\varphi-\omega s)$ dominates the $\mathrm{O}\left(\mu^{2}\right)$-term, one has to assume $\mu$ exponentially small with respect to $\varepsilon$. For larger values of $\mu$, the existence of intersections cannot follow directly from (14).

In fact, the study of the splitting in the singular case requires a more careful analysis, ${ }^{6,7,8}$ which is not carried out here. Nevertheless, the effective existence of a number of homoclinic intersections, for both the regular and singular cases, will be established in section 6 .

\section{Some examples with small divisors}

To illustrate the properties of the Melnikov potential $L$, in this section we consider some examples, showing that $L$ has nondegenerate critical points. As a measure of the transversality of these points as zeros of $M=\partial_{\varphi} L$, we also estimate the determinant of the symmetric matrix $\partial_{\varphi} M=\partial_{\varphi}^{2} L$ at the critical points.

Consider the Hamiltonian $H=H_{0}+\mu H_{1}$, with

$$
\begin{aligned}
& H_{0}(x, y, I)=\langle\omega, I\rangle+\frac{1}{2}\langle\Lambda I, I\rangle+\frac{y^{2}}{2}+\cos x-1, \\
& H_{1}(x, \varphi)=(\cos x-1) f(\varphi), \quad f(\varphi)=\sum_{k \in \mathbb{Z}^{n}} f_{k} e^{i\langle k, \varphi\rangle} .
\end{aligned}
$$

The integrable Hamiltonian $H_{0}$ is uncoupled $(\lambda=0$ in (2)), and consists of a pendulum and $n$ rotors (the standard pendulum is given by $V(x)=\cos x-1$ ). Note that the perturbation $H_{1}$ depends only on the angles $x, \varphi$ and that, since $H_{1}=\mathrm{O}_{2}(x)$, the whiskered torus remains fixed.

We do not assume that $f(\varphi)$ is a trigonometric polynomial, but rather that it includes harmonics in $\varphi$ of arbitrarily high orders. If the Fourier coefficients of the function $f(\varphi)$ are exponentially decreasing:

$$
\left|f_{k}\right| \sim e^{-|k| \rho},
$$

then this is an analytic function, and $\rho$ is its width of analyticity in the angles $\varphi$. On the contrary, if the coefficients are polynomially decreasing:

$$
\left|f_{k}\right| \sim \frac{1}{|k|^{r}},
$$


then the function $f(\varphi)$ is not analytic but only differentiable (it is $\mathcal{C}^{p}$ for any $p<r-n)$.

Since we are interested in a singular situation, we consider fast frequencies $\omega=\omega^{*} / \sqrt{\varepsilon}$. We assume that the vector $\omega^{*}$ is Diophantine, and introduce $\gamma=\gamma^{*} / \sqrt{\varepsilon}$ in (6), for some $\tau \geq n-1$.

\section{Melnikov coefficients}

The fact that $H_{1}=\mathrm{O}_{2}(x)$ allows us to compute the Melnikov potential applying the simple formula (11). In the integral, we have to consider the wellknown (positive) homoclinic trajectory of the standard pendulum:

$$
x_{0}(t)=4 \arctan e^{t}, \quad y_{0}(t)=\dot{x}_{0}(t)=\frac{2}{\cosh t} .
$$

We have:

$L(\varphi)=-\int_{-\infty}^{\infty}\left(\cos x_{0}(t)-1\right) f(\varphi+\omega t) \mathrm{d} t+$ const $=2 \int_{-\infty}^{\infty} \frac{f(\varphi+\omega t)}{\cosh ^{2} t} \mathrm{~d} t+$ const.

Taking into account that the additive constant is such that $\bar{L}=0$, and writing $L(\varphi)=\sum_{k \neq 0} L_{k} e^{i\langle k, \varphi\rangle}$, the Fourier coefficients $L_{k}$ can be computed explicitly using residue theory:

$$
L_{k}=2 f_{k} \int_{-\infty}^{\infty} \frac{e^{i\langle k, \omega\rangle t}}{\cosh ^{2} t} \mathrm{~d} t=\frac{2 \pi\langle k, \omega\rangle f_{k}}{\sinh \left(\frac{\pi}{2}\langle k, \omega\rangle\right)}, \quad k \neq 0
$$

(note that the mean value $\bar{f}=f_{0}$ does not influence the Melnikov potential). For the Melnikov function, it is clear that $M_{k}=i k L_{k}$.

\section{Upper bounds}

In the analytic case (15), an upper bound for the Melnikov potential $L$ can be given ${ }^{10}$ from the expressions of the coefficients. The upper bound obtained holds for $\varepsilon>0$ small enough, and is exponentially small in $\varepsilon$. Its size depends strongly on the small divisors properties of the frequencies:

$$
\max _{\varphi \in \mathbb{T}^{n}}|L(\varphi)| \leq \frac{\text { const }}{\varepsilon^{1 /(2 \tau+2)}} \exp \left(-C \varepsilon^{-1 /(2 \tau+2)}\right),
$$

where $C=C\left(\tau, \gamma^{*}, \rho\right)$ is a constant. It is an important point in this estimate to assume a perturbation with an infinite number of harmonics. As stressed by Lochak, ${ }^{4}$ one is then forced to take into account the small divisors associated to the frequencies, and this leads to the exponent $1 /(2 \tau+2)$ inside the exponential. Notice that this exponent in the upper bound is reminiscent 
of the Nekhoroshev-like estimates. Instead, if one assumes a finite number of harmonics (like in the Arnold's example ${ }^{5}$ ), then one obtains the exponent $1 / 2$, but this case is highly nongeneric.

In an analogous way, we can obtain an upper bound for the differentiable case (16), but then the bound becomes a power of $\varepsilon$ :

$$
\max _{\varphi \in \mathbb{T}^{n}}|L(\varphi)| \leq \text { const } \cdot \varepsilon^{r / 2 \tau} .
$$

The golden mean and the Fibonacci numbers

To establish the effective existence of splitting, one has to obtain also lower bounds, giving a more precise description of the asymptotic behavior of the Melnikov potential. This requires a more careful analysis of the small divisors associated to the frequency vector $\omega^{*}$. This analysis can easily be carried out for the golden mean, a very simple case with 2 frequencies (i.e. with 3 degrees of freedom):

$$
\omega^{*}=(1, \Omega), \quad \Omega=\frac{\sqrt{5}+1}{2} .
$$

This case was first considered by Simó ${ }^{17}$ and, later on, ${ }^{6}$ lower bounds for the Melnikov function and for the splitting, in the analytic case (15), were obtained. Recently, ${ }^{10}$ lower bounds have been obtained for the Melnikov potential $L$ and for the determinant of $\partial_{\varphi}^{2} L$ at the critical points (ensuring also that $L$ has nondegenerate critical points). These lower bounds (recalled in the second example below) are exponentially small with respect to $\varepsilon$. This implies that, in order to deduce the existence of splitting as a consequence of theorem 2, the parameter $\mu$ has to be taken exponentially small in $\varepsilon$.

The differentiable case (16) is substantially different. A concrete example ${ }^{18}$ shows that the maximum of the Melnikov function has a lower bound of finite order in $\varepsilon$. Then taking $\mu$ as a suitable power of $\varepsilon$ is enough in order to establish the existence of splitting from theorem 2.

We stress that an essential point in dealing with the singular case is to assume that, in the perturbation, at least the harmonics $f_{k}$ corresponding to the small divisors associated to $\omega^{*}$ are nonvanishing, because the dominant harmonic is found among these ones. Under this assumption, one can obtain ${ }^{6}$ the largest lower bounds in the Melnikov approximation, in order to ensure that this approximation dominates the $\mathrm{O}\left(\mu^{2}\right)$-remainder.

For the golden mean (19), the associated small divisors are directly related to the Fibonacci numbers:

$$
F_{0}=F_{1}=1, \quad F_{n}=F_{n-1}+F_{n-2}, \quad n \geq 2 .
$$


We recall some basic facts concerning these numbers, that will be used below. Defining

$$
C_{F}=\frac{1}{\Omega+\Omega^{-1}}=\frac{1}{\sqrt{5}}
$$

we have

$$
F_{n}=C_{F}\left(\Omega^{n+1}-(-1)^{n+1} \Omega^{-(n+1)}\right), \quad n \geq 0 .
$$

The best rational approximations of $\Omega$ are given by the convergents $F_{n} / F_{n-1}$. In other words, the indexes $k^{(n)}=\left(F_{n},-F_{n-1}\right)$ (and also $\left(-F_{n}, F_{n-1}\right)$ ) are the ones that give the dominant behavior among the small divisors $\left\langle k, \omega^{*}\right\rangle$. More precisely, one has:

$$
\left\langle k^{(n)}, \omega^{*}\right\rangle=F_{n}-F_{n-1} \Omega=\frac{(-1)^{n}}{\Omega^{n}}=\frac{(-1)^{n} C_{F}}{F_{n-1}}+\mathrm{O}\left(\frac{1}{F_{n-1}^{3}}\right), \quad n \geq 1,
$$

and also the following inequality: ${ }^{6}$ for any $k=\left(k_{1},-k_{2}\right)$ such that $k_{2}>0$ is not a Fibonacci number,

$$
\left|\left\langle k, \omega^{*}\right\rangle\right|=\left|k_{1}-k_{2} \Omega\right|>\frac{\Omega C_{F}}{k_{2}} .
$$

Note that the frequency vector (19) satisfies the Diophantine condition (6) with $\tau=1$. This frequency vector is considered in the two examples that we next study.

Lower bounds: An example with finite-order splitting

Now we consider a concrete example in the differentiable case (16), analogous to the one of Delshams et al., ${ }^{18}$ and obtain lower bounds for the maximum of the Melnikov potential $L$, and for the determinant of $\partial_{\varphi}^{2} L$ at a critical point.

For the perturbation, we consider the following function:

$$
f(\varphi)=\sum_{n \geq 1} \frac{1}{\Omega^{n r}} \cos \left\langle k^{(n)}, \varphi\right\rangle .
$$

In this function, the only nonvanishing Fourier coefficients are the ones associated to the Fibonacci indexes $k^{(n)}$. Since $\left|k^{(n)}\right|=F_{n+1} \sim \Omega^{n+2}$, the coefficients decrease as in (16).

Applying (17), the Melnikov potential $L(\varphi)$ is given by the series

$$
L(\varphi)=\sum_{n \geq 1} S_{n} \cos \left\langle k^{(n)}, \varphi\right\rangle
$$


with

$$
S_{n}=\frac{2 \pi}{\sqrt{\varepsilon}} \cdot \frac{1}{\Omega^{n(r+1)} \sinh \left(\frac{\pi}{2 \Omega^{n} \sqrt{\varepsilon}}\right)} .
$$

Note that all the coefficients are positive. The main part of this expression is given by

$$
S_{n}^{0}=\frac{4 \pi}{\sqrt{\varepsilon}} e^{-b_{n}^{0}}, \quad b_{n}^{0}=b_{n}^{0}(\varepsilon)=n(r+1) \log \Omega+\frac{\pi}{2 \Omega^{n} \sqrt{\varepsilon}} .
$$

To determine the dominant behavior, we look for the minimum exponent $b_{n}^{0}$, for $n \geq 1$. This is reached for $N_{0}=N_{0}(\varepsilon)$, with

$$
\Omega^{N_{0}}=\frac{\pi}{2(r+1) \sqrt{\varepsilon}}
$$

and this gives the largest coefficient: $S_{N_{0}} \geq S_{N_{0}}^{0} \sim \varepsilon^{r / 2}$. This coefficient itself constitutes a lower bound for the maximum of the Melnikov potential, because all the coefficients are positive and

$$
\max _{\varphi \in \mathbb{T}^{2}}|L(\varphi)|=L(0)=\sum_{n \geq 1} S_{n}
$$

We can also get an upper bound, which coincides with the one of (18). Let us break the series (21) in two parts. For $n \leq N_{0}$, note that

$$
\begin{aligned}
\frac{S_{n}^{0}}{S_{n-1}^{0}} & =\frac{1}{\Omega^{r+1}} \exp \left(\frac{\pi}{2 \Omega^{n+1} \sqrt{\varepsilon}}\right) \\
& \geq \frac{1}{\Omega^{r+1}} \exp \left(\frac{\pi}{2 \Omega^{N_{0}+1} \sqrt{\varepsilon}}\right)=\left(\frac{e^{1 / \Omega}}{\Omega}\right)^{r+1}>1 .
\end{aligned}
$$

Using also $S_{n} \leq \frac{3}{2} S_{n}^{0}$ (from the fact that $\sinh x \geq e^{x} / 3$ for $x \geq 1$ ), the sum $\sum_{n \leq N_{0}} S_{n}$ has an upper bound of the same order as $S_{N_{0}}$. On the other hand, for $n>N_{0}$ we have the inequality $S_{n} \leq 4 \Omega^{-n r}$ (simply using that $\sinh x \geq x$ ), and we can bound $\sum_{n>N_{0}} S_{n}$ as a geometric series. In this way, we obtain for the maximum value of the Melnikov potential, an upper bound and a lower bound, both of the same order:

$$
\max _{\varphi \in \mathbb{T}^{2}}|L(\varphi)| \sim \varepsilon^{r / 2}
$$

The Melnikov potential $L(\varphi)$ has $\varphi^{*}=0$ as a critical point. Now, we want to show that this critical point is nondegenerate, estimating also the 
eigenvalues of $\partial_{\varphi}^{2} L(0)$ as a measure for the transversality. We have

$$
\partial_{\varphi}^{2} L(0)=-\sum_{n \geq 1} S_{n} k^{(n)}\left(k^{(n)}\right)^{\top}
$$

and then

$$
\begin{aligned}
\operatorname{det} \partial_{\varphi}^{2} L(0) & =\left(\sum_{n \geq 1} F_{n}^{2} S_{n}\right)\left(\sum_{n \geq 1} F_{n-1}^{2} S_{n}\right)-\left(\sum_{n \geq 1} F_{n} F_{n-1} S_{n}\right)^{2} \\
& =\sum_{n, m \geq 1} F_{n} F_{m-1}\left(F_{n} F_{m-1}-F_{n-1} F_{m}\right) S_{n} S_{m} \\
& =\sum_{1 \leq m<n}\left(F_{n} F_{m-1}-F_{n-1} F_{m}\right)^{2} S_{n} S_{m}=\sum_{1 \leq m<n} F_{n-m-1}^{2} S_{n} S_{m}
\end{aligned}
$$

where we have used the formula $F_{n} F_{m-1}-F_{n-1} F_{m}=(-1)^{m+1} F_{n-m-1}$. Note that all terms in this series are also positive. To estimate the determinant, note that

$$
F_{n-m-1}^{2} S_{n} S_{m} \sim \Omega^{2 n} S_{n} \cdot \Omega^{-2 m} S_{m},
$$

and hence the indexes $n$ and $m$ can be separated. This allows us to find the indexes $N_{1}(\varepsilon)$ and $M_{1}(\varepsilon)$ that give the dominant term in the series of the determinant, in the same way as before. In this way, we easily obtain an upper bound and a lower bound for the determinant:

$$
\operatorname{det} \partial_{\varphi}^{2} L(0) \sim \varepsilon^{r}
$$

In fact, we should estimate the minimum eigenvalue of $\partial_{\varphi}^{2} L(0)$. This eigenvalue can be put in terms of $\tau=\operatorname{tr} \partial_{\varphi}^{2} L(0)$ and $\delta=\operatorname{det} \partial_{\varphi}^{2} L(0)$. Again, note that $\tau \sim \varepsilon^{r / 2}$ (applying the same method). The minimum eigenvalue is given by

$$
\frac{\tau-\sqrt{\tau^{2}-4 \delta}}{2} \geq \frac{\delta}{\tau}
$$

and this has clearly a lower bound of order $\varepsilon^{r / 2}$. Then it is a consequence of theorem 2 that, for $\mu=o\left(\varepsilon^{r / 2}\right)$, the critical point $\varphi^{*}=0$ of $L(\varphi)$ gives rise to a transverse homoclinic orbit. This result makes a difference with the second example, next considered, and is due to the non-analyticity of the perturbation. 
Lower bounds: A singular example

For the sake of completeness, we also include an example ${ }^{10}$ in the analytic case (16). For the perturbation, we consider a "full" Fourier series, with the coefficients

$$
\left|f_{k}\right|=e^{-|k| \rho} \quad \forall k \in \mathbb{Z}^{2} \backslash\{0\} .
$$

Note that a non-even function $f(\varphi)$ is allowed, so we are not assuming that the perturbation $H_{1}(x, \varphi)$ is reversible, unlike other papers. ${ }^{19,7,8}$

Following the method by Delshams et al. ${ }^{6}$ (though the context is somewhat different), it is shown ${ }^{10}$ that the dominant harmonics in the Fourier series of the Melnikov potential $L(\varphi)$ are the ones associated to the Fibonacci indexes $k^{(n)}$. Denoting $S_{n}=L_{k^{(n)}}$, from (17) one directly obtains

$$
\left|S_{n}\right|=\frac{2 \pi}{\Omega^{n} \sqrt{\varepsilon}} \cdot \frac{e^{-F_{n+1} \rho}}{\sinh \left(\frac{\pi}{2 \Omega^{n} \sqrt{\varepsilon}}\right)}, \quad n \geq 1 .
$$

The main part of this expression is given by

$$
\left|S_{n}^{0}\right|=\frac{4 \pi}{\Omega^{n} \sqrt{\varepsilon}} e^{-b_{n}^{0}}, \quad b_{n}^{0}=b_{n}^{0}(\varepsilon)=C_{F} \Omega^{n+2} \rho+\frac{\pi}{2 \Omega^{n} \sqrt{\varepsilon}} .
$$

For a fixed $\varepsilon>0$, to find the dominant harmonic among the Fibonacci ones, one has to look for the minimum exponent $b_{n}^{0}, n \geq 1$. Let us define

$$
\varepsilon_{n}=\left(\frac{D_{0}}{\Omega^{n+1}}\right)^{4}=\frac{\varepsilon_{0}}{\Omega^{4 n}}, \quad D_{0}=\sqrt{\frac{\pi}{2 C_{F} \rho}} .
$$

The minimum exponent among the $b_{n}^{0}$ is reached for an only integer $N_{0}=$ $N_{0}(\varepsilon)$, such that $\log \varepsilon_{N_{0}}$ is the closest to $\log \varepsilon$, among the $\log \varepsilon_{n}$. Then the coefficient $S_{N_{0}}^{0}$ is the dominant one among the $S_{n}^{0}$, and it is not hard to check that the "whole" coefficient $S_{N_{0}}$ is also dominant among the $S_{n}$. One can also check from (20) that the non-Fibonacci coefficients $L_{k}$, with $k \neq k^{(n)}$, do not dominate. In terms of $\varepsilon$, the value of the minimum exponent depends on $\varepsilon$ in the following way:

$$
b_{N_{0}}^{0}=\frac{c(\log \varepsilon)}{\varepsilon^{1 / 4}}
$$

where $c(\eta)$ is a continuous function, defined as the $(4 \log \Omega)$-periodic extension of

$$
c(\eta)=C_{0} \cosh \left(\frac{\eta-\eta_{0}}{4}\right), \quad\left|\eta-\eta_{0}\right| \leq 2 \log \Omega,
$$


with

$$
C_{0}=\Omega \sqrt{2 \pi C_{F} \rho}, \quad \eta_{0}=\log \varepsilon_{0} .
$$

The extreme values of this function are given by

$$
C_{0} \leq c(\eta) \leq \frac{\Omega^{3 / 2} C_{0}}{2}=(1.029085 \ldots) C_{0} .
$$

In this way, the maximum value of the Melnikov potential can be approximated by its dominant Fibonacci harmonic, and one obtains the following upper and lower bound:

$$
\max _{\varphi \in \mathbb{T}^{2}}|L(\varphi)| \sim \frac{1}{\varepsilon^{1 / 4}} \exp \left(-\frac{c(\log \varepsilon)}{\varepsilon^{1 / 4}}\right) .
$$

It is also shown ${ }^{10}$ that the Melnikov potential $L(\varphi)$ has nondegenerate critical points. In order to detect these points, one has to consider an approximation given by at least the 2 dominant harmonics, because with only 1 harmonic the approximation to the matrix $\partial_{\varphi}^{2} L$ would be degenerate. In the discussion above, it can also be considered the integer $N_{1}(\varepsilon)$ reaching the "second" minimum among the $b_{n}^{0}$; this integer satisfies $\left|N_{1}-N_{0}\right|=1$. Calling $N=N(\varepsilon)=\min \left(N_{0}, N_{1}\right)$, it turns out that $\varepsilon_{N+1}<\varepsilon<\varepsilon_{N}$, and the Fibonacci coefficients with indexes $N$ and $N+1$ give the 2 dominant harmonics in the Fourier expansion of the Melnikov potential.

The two dominant harmonics give the main part of the Melnikov potential $L(\varphi)$. In the trigonometric form, this main part can be written as

$$
L(\varphi) \sim 2\left|S_{N}\right| \cos \left(\left\langle k^{(N)}, \varphi\right\rangle+\sigma_{N}\right)+2\left|S_{N+1}\right| \cos \left(\left\langle k^{(N+1)}, \varphi\right\rangle+\sigma_{N+1}\right),
$$

where $\sigma_{N}, \sigma_{N+1}$ are some phases. The number of critical points is given by the determinant

$$
\Delta_{N}=\operatorname{det}\left(k^{(N)}, k^{(N+1)}\right)=F_{N-1} F_{N+1}-F_{N}^{2}=(-1)^{N+1},
$$

which implies that, for $\varepsilon$ small enough, $L(\varphi)$ has exactly 4 critical points. At every critical point $\varphi^{*}$, one has

$$
\left|\operatorname{det} \partial_{\varphi}^{2} L\left(\varphi^{*}\right)\right| \sim 4\left|S_{N} S_{N+1}\right| \neq 0
$$

Then $L(\varphi)$ is a Morse function, because all its critical points are nondegenerate. Note also that $4=2^{2}$ is the minimum number of critical points for a Morse function on $\mathbb{T}^{2}$. To estimate the size of the determinant, we use (22) again:

$$
4\left|S_{N}^{0} S_{N+1}^{0}\right|=\frac{64 \pi^{2}}{\Omega^{2 N+1} \varepsilon} e^{-\left(b_{N}^{0}+b_{N+1}^{0}\right)}, \quad b_{N}^{0}+b_{N+1}^{0}=\frac{c_{1}(\log \varepsilon)}{\varepsilon^{1 / 4}},
$$


where $c_{1}(\eta)$ is another $(4 \log \Omega)$-periodic function, defined from

$$
c_{1}(\eta)=\Omega^{3 / 2} C_{0} \cosh \left(\frac{\eta-\eta_{0}^{\prime}}{4}\right), \quad\left|\eta-\eta_{0}^{\prime}\right| \leq 2 \log \Omega
$$

with $\eta_{0}^{\prime}=\log \sqrt{\varepsilon_{0} \varepsilon_{1}}$. The extreme values of this function are given by

$$
\Omega^{3 / 2} C_{0} \leq c_{1}(\eta) \leq \frac{\Omega^{3} C_{0}}{2}
$$

Thus, one obtains an upper bound and a lower bound for the determinant at the 4 critical points:

$$
\operatorname{det} \partial_{\varphi}^{2} L\left(\varphi^{*}\right) \sim \frac{1}{\varepsilon^{1 / 2}} \exp \left(-\frac{c_{1}(\log \varepsilon)}{\varepsilon^{1 / 4}}\right) .
$$

Proceeding as in the previous example, one also finds an estimate for the minimum eigenvalue. Since, in this case, $\delta \ll \tau$, the minimum eigenvalue can be approximated by $\delta / \tau$. This leads to an estimate of the type

$$
\frac{1}{\varepsilon^{1 / 4}} \exp \left(-\frac{c_{2}(\log \varepsilon)}{\varepsilon^{1 / 4}}\right)
$$

where $c_{2}(\eta)=c_{1}(\eta)-c(\eta)$. This is also a positive periodic function, with

$$
\frac{\Omega^{3 / 2} C_{0}}{2} \leq c_{2}(\eta) \leq\left(\Omega-\frac{1}{2}\right) C_{0}
$$

Then it is a direct consequence of theorem 2 that there exist 4 transverse homoclinic intersections, for $\mu=o\left(\exp \left\{-c_{2}(\log \varepsilon) \varepsilon^{-1 / 4}\right\}\right)$. The estimate obtained gives a measure for the transversality of the splitting. However, it has to be recalled again that this is actually a regular situation, and a justification in the singular case $\mu=\varepsilon^{p}$, for some $p>0$, does not follow directly from theorem 2 .

\section{Flow-box variables and splitting potential}

The aim of this section is to sketch the proof of the result ${ }^{10}$ that, using suitable variables, the "whole" splitting distance (and not only its first order approximation) is the gradient of some function, in order to establish the existence of homoclinic orbits even in the singular case. 
Flow-box variables

In order to provide a clearer formulation for the problem of measuring the splitting, it is convenient to introduce new symplectic variables in which the Hamiltonian equations are very simple. The flow-box variables $W=$ $(S, E, \psi, J)$ are constructed ${ }^{10}$ with the help of the flow associated to the normal form $\widetilde{H}$ given in (8), from a suitable Poincare section containing the set $\mathcal{S}=\tilde{z}\left(s_{1}, \mathbb{T}^{n}\right) \subset \widetilde{\mathcal{W}}_{\text {loc }}^{+}$, with some fixed $s_{1}>s_{0}$. The new variables are then given by an exact symplectic transformation $(x, y, \varphi, I)=\Psi(S, E, \psi, J)$, defined on a real neighborhood of $\widehat{\mathcal{S}}=\Psi^{-1}(\mathcal{S})=\left(0,0, \mathbb{T}^{n}, a\right)$.

Thanks to the use of the Kolmogorov's approach to the hyperbolic KAM theory, the neighborhood where the flow-box variables are defined contains a piece of both whiskers. In the construction of the variables, one can make the local stable whisker become a coordinate plane (see (24)), and then the global unstable whisker can be seen as a graphic over the local stable one. In this way, the splitting distance and the homoclinic intersections between the two whiskers appear much more transparently.

Our Hamiltonian takes, in the flow-box variables, a very simple form:

$$
\widehat{H}=\widetilde{H} \circ \Psi=E+\langle\omega, J-a\rangle,
$$

and hence the associated Hamiltonian equations are

$$
\dot{S}=1, \quad \dot{E}=0, \quad \dot{\psi}=\omega, \quad \dot{J}=0 .
$$

We recall that analogous flow-box variables have already been used ${ }^{20,6}$ in some case where the symplectic change can be defined explicitly from the expression of the normal form, which is integrable. In our case, the normal form $\widetilde{H}$ is, in general, not integrable, and the construction of the flow-box variables is more involved (it uses implicit functions).

Parameterizations in the flow-box variables

Let us describe more precisely how the whiskers can be parameterized in the flow-box variables. Let us denote $\widehat{\mathcal{W}}_{\text {loc }}^{+}=\Psi^{-1}\left(\widetilde{\mathcal{W}}_{\text {loc }}^{+}\right)$the local stable whisker (or more precisely a piece of it). This whisker becomes a coordinate plane, given by $E=0, J=a$, and can be parameterized as follows:

$$
\widehat{\mathcal{W}}_{\text {loc }}^{+}: \quad W_{\text {loc }}^{+}(s, \varphi)=\Psi^{-1}\left(\tilde{z}\left(s_{1}+s, \varphi\right)\right)=(s, 0, \varphi, a), \quad s \in \mathcal{I}, \quad \varphi \in \mathbb{T}^{n},
$$

where $\mathcal{I}$ is some interval containing $s=0$ (we have replaced $s-s_{1}$ by $s$ for a clearer notation: in this way we have $s=0$ on $\widehat{\mathcal{S}}$ ). 
Now, we define $\widehat{\mathcal{W}}^{-}=\Psi^{-1} \circ \Phi^{-1}\left(\mathcal{W}^{-}\right)$as an invariant manifold of $\widehat{H}$, which is the equivalent in the flow-box variables for (a piece of) the global unstable whisker. Let us parameterize:

$$
\widehat{\mathcal{W}}^{-}: \quad W^{-}(s, \varphi)=\Psi^{-1} \circ \Phi^{-1}\left(z^{-}(s, \varphi)\right), \quad s \in \mathcal{I}, \quad \varphi \in \mathbb{T}^{n} .
$$

In components, we write

$$
W^{-}(s, \varphi)=\left(S^{-}(s, \varphi), E^{-}(s, \varphi), \psi^{-}(s, \varphi), J^{-}(s, \varphi)\right) .
$$

There is splitting of the whiskers when $J^{-}(s, \varphi) \neq a$ or $E^{-}(s, \varphi) \neq 0$. Nevertheless, it suffices to control the $J$-component because the whisker is contained in the zero energy level: $E^{-}+\left\langle\omega, J^{-}-a\right\rangle=0$.

The approximation given in theorem 2, expressed there in the original variables, remains true after changing to the flow-box variables. So the Melnikov function $M=\partial_{\varphi} L$ also provides a first order approximation in $\mu$ for the splitting distance $J^{-}(s, \varphi)-a$, at least in the regular case. But, as a new feature, in the flow-box variables this splitting distance becomes a quasiperiodic function, only depending on $\varphi-\omega s$. Indeed, from the simple form (23) of the Hamiltonian equations, one sees that

$$
J^{-}(s, \varphi)=J^{-}(0, \varphi-\omega s) .
$$

This property is very important in the singular case, because it can give rise to exponentially small estimates for the splitting. The key point ${ }^{6,20}$ is to use that a function of $\varphi-\omega s$, with $\omega=\omega^{*} / \sqrt{\varepsilon}$, having a polynomial bound on a complex domain, becomes exponentially small in $\varepsilon$ for real values of $s, \varphi$.

\section{Splitting potential and existence of homoclinic orbits}

Using the special formulation of theorem 1 (in the original variables) it is possible ${ }^{1}$ to introduce in some neighborhood an exact symplectic map $\Theta$ that takes the local stable whisker onto the global unstable one. In fact, one can go a bit farther, ${ }^{10}$ and express the map $\Theta$ in the flow-box variables.

The exactness of the symplectic map $\Theta$ and the fact that $\Theta=$ id + $\mathrm{O}(\mu)$, imply that for $|\mu|$ small enough there exists a generating function $\theta(S, \check{E}, \psi, \check{J})$, defined in some neighborhood of $\widehat{\mathcal{S}}$ and global in the angles $\psi \in \mathbb{T}^{n}$, such that the map $\Theta:(\breve{S}, \check{E}, \breve{\psi}, \breve{J}) \mapsto(S, E, \psi, J)$ is given by

$$
S=\check{S}-\partial_{\check{E}} \theta, \quad \check{E}=E-\partial_{S} \theta, \quad \psi=\check{\psi}-\partial_{\breve{J}} \theta, \quad \check{J}=J-\partial_{\psi} \theta
$$

In order to compare the whiskers $\widehat{\mathcal{W}}_{\text {loc }}^{+}$and $\widehat{\mathcal{W}}^{-}$, it will be useful to express the splitting distance $J^{-}(s, \varphi)-a$ as a gradient. This cannot be deduced 
directly from (25), but this obstruction is easily overcome, ${ }^{20}$ introducing new parameters that substitute the initial ones $s, \varphi$ on the whiskers:

$$
S=S^{-}(s, \varphi), \quad \psi=\psi^{-}(s, \varphi) .
$$

In terms of the new parameters $S, \psi$, the unstable whisker $\widehat{\mathcal{W}}^{-}$appears nicely as a graphic over the stable whisker $\widehat{\mathcal{W}}_{\text {loc }}^{+}$, through the parameterization:

$$
\widehat{\mathcal{W}}^{-}: \quad \tilde{W}^{-}(S, \psi)=\left(S, \tilde{E}^{-}(S, \psi), \psi, \tilde{J}^{-}(S, \psi)\right), \quad S \in \mathcal{I}, \quad \psi \in \mathbb{T}^{n}
$$

(the interval $\mathcal{I}$ can have undergone a reduction).

It is then natural to introduce the splitting potential as the following scalar function, periodic in $\psi$ :

$$
\mathcal{L}(S, \psi)=\theta(S, 0, \psi, a), \quad S \in \mathcal{I}, \quad \psi \in \mathbb{T}^{n} .
$$

This function also depends on $\mu$, and is determined up to an additive constant. The (vector) splitting function can then be defined as the gradient of $\mathcal{L}$ with respect to the angles:

$$
\mathcal{M}(S, \psi)=\partial_{\psi} \mathcal{L}(S, \psi) .
$$

The next theorem is easily deduced from the equations (25).

Theorem 3 The functions $\mathcal{L}$ and $\mathcal{M}$ only depend on $\psi-\omega S$ :

$$
\mathcal{L}(S, \psi)=\mathcal{L}(0, \psi-\omega S), \quad \mathcal{M}(S, \psi)=\mathcal{M}(0, \psi-\omega S) .
$$

Besides, these functions are related with (24) and (26) in the following way:

$$
\tilde{E}^{-}(S, \psi)=\partial_{S} \mathcal{L}(S, \psi), \quad \tilde{J}^{-}(S, \psi)-a=\mathcal{M}(S, \psi) .
$$

According to this theorem, the function $\mathcal{M}$ gives the splitting distance (expressed in the parameters $S, \psi$ ). It is important to stress that the fact that the splitting distance can be put as the gradient of some potential is a reflection of the Lagrangian properties of the whiskers.

As a corollary of theorem 3, one can recover a result due to Eliasson: ${ }^{1}$ there exist at least $n+1$ homoclinic orbits (not necessarily transverse), biasymptotic to the whiskered torus $\mathcal{T}$. This result, valid for both the regular case and the singular case, comes from the fact that a function on $\mathbb{T}^{n}$ has at least $n+1$ critical points (not necessarily nondegenerate), according to the Lyusternik-Schnirelman theory. ${ }^{21}$ Then for a fixed $S$, the splitting potential $\mathcal{L}(S, \cdot)$ has at least $n+1$ critical points, which give rise to respective homoclinic intersections between the whiskers $\mathcal{W}^{ \pm}$, and hence to homoclinic orbits, contained in both whiskers. 
First order approximation for the splitting potential

Finally, using Poincaré-Melnikov theory, we can obtain first order approximations for the splitting potential $\mathcal{L}$, introduced in $(27)$, and for the splitting function $\mathcal{M}$. At first order in $\mu$, these approximations are given, respectively, by the Melnikov potential $L$ and the Melnikov function $M$ defined in section 4 , but they are good enough only for the regular case.

Theorem 4 For $S \in \mathcal{I}$ and $\psi \in \mathbb{T}^{n}$, one has

$$
\mathcal{L}(S, \psi)=\mu L(\psi-\omega S)+\mathrm{O}\left(\mu^{2}\right), \quad \mathcal{M}(S, \psi)=\mu M(\psi-\omega S)+\mathrm{O}\left(\mu^{2}\right) .
$$

We finish with some remarks about the additional difficulties of the singular case. Note that theorem 4 provides an $\mathrm{O}\left(\mu^{2}\right)$ error term that is not small enough in the singular case $\mu=\varepsilon^{p}$ with $p>0$, due to the fact that the functions $L$ and $M$ are exponentially small with respect to $\varepsilon$. (This is illustrated in the second example of section 5). Nevertheless, one can expect that, under some weak hypotheses on the perturbation, the predictions of the splitting given by the Melnikov potential $L$ are also valid in the singular case, for some $p>0$.

To get better bounds of the $\mathrm{O}\left(\mu^{2}\right)$-term for real values of the variables $S, \psi$, one should bound this term on a complex strip of these variables. This requires some improvements of the results presented here. First, one needs a more precise version of theorem 1 , carrying out a careful control on the loss $\delta$ of complex domain in the angular variables. Such an improvement of the normal form theorem has already been performed by the authors, ${ }^{10}$ and in fact analogous results had previously been obtained ${ }^{2,6}$ for somewhat different contexts.

On the other hand, one needs an extension theorem and the flow-box variables extended to a suitable complex domain, which would lead to a significant refinement of theorem 4 , of the type

$$
\mathcal{L}(S, \psi)=\mu L\left(\psi-S \omega^{*} / \sqrt{\varepsilon}\right)+\mathrm{O}\left(\mu^{2} \varepsilon^{-p}\right),
$$

for $S, \psi$ on a complex strip $|\operatorname{Im} S| \leq \pi / 2-\varepsilon^{1 / 4},|\operatorname{Im} \psi| \leq \rho-\varepsilon^{1 / 4}$. Then one could obtain, for real values of $S, \psi$, exponentially small upper bounds for the error term, which would be dominated by the first order approximation provided by Poincaré-Melnikov theory, under some general hypotheses on the perturbation. If this is true, then the Poincaré-Melnikov theory gives the right predictions for the splitting even in the singular case.

The problem of giving asymptotics for the exponentially small splitting of separatrices is now being researched by the authors. In fact, the strategy described above has been followed ${ }^{20,6}$ in simpler situations in which the normal 
form is integrable and the flow-box variables can be defined explicitly. Rudnev and Wiggins ${ }^{8}$ announced an important generalization, but their proof contains essential errors. Therefore, the problem of giving asymptotics for the splitting in the Hamiltonian (1-2), in the singular case, remains still open.

\section{Acknowledgments}

This work has been supported in part by the Catalan grant CIRIT 1998SGR00042. Research by A.D. is also supported by the Spanish grant DGICYT PB94-0215.

\section{References}

1. L.H. Eliasson. Biasymptotic solutions of perturbed integrable Hamiltonian systems. Bol. Soc. Bras. Mat, 25(1):57-76, 1994.

2. M. Rudnev and S. Wiggins. KAM theory near multiplicity one resonant surfaces in perturbations of a-priori stable Hamiltonian systems. $J$. Nonlinear Sci., 7:177-209, 1997.

3. A. Delshams and P. Gutiérrez. Homoclinic orbits to invariant tori in Hamiltonian systems. In C. Jones, S. Wiggins, A. Khibnik, F. Dumortier, and D. Terman, editors, Multiple-Time-Scale Dynamical Systems, IMA Volumes in Mathematics and Its Applications. Held in Minneapolis, 2731 October, 1997. Springer-Verlag, to appear in 1999.

4. P. Lochak. Canonical perturbation theory via simultaneous approximation. Russian Math. Surveys, 47(6):57-133, 1992.

5. V.I. Arnold. Instability of dynamical systems with several degrees of freedom. Soviet Math. Dokl., 5(3):581-585, 1964.

6. A. Delshams, V.G. Gelfreich, À. Jorba, and T.M. Seara. Exponentially small splitting of separatrices under fast quasiperiodic forcing. Comm. Math. Phys., 189:35-71, 1997.

7. G. Gallavotti, G. Gentile, and V. Mastropietro. Pendulum: separatrix splitting. Preprint 97-472, mp_arc@math . utexas . edu, 1997.

8. M. Rudnev and S. Wiggins. Existence of exponentially small separatrix splitting and homoclinic connections between whiskered tori in weakly hyperbolic near-integrable Hamiltonian systems. Phys. D, 114:3-80, 1998.

9. S.V. Bolotin. Homoclinic orbits in invariant tori of Hamiltonian systems. In V.V. Kozlov, editor, Dynamical systems in classical mechanics, volume 168 of Amer. Math. Soc. Transl. Ser. 2, pages 21-90. Amer. Math. Soc., Providence, RI, 1995. (Adv. Math. Sci., 25). 
10. A. Delshams and P. Gutiérrez. Splitting potential and PoincaréMelnikov theory for whiskered tori in Hamiltonian systems. Preprint, submitted to J. Nonlinear Sci., 1998.

11. L. Niederman. Dynamics around simple resonant tori in nearly integrable Hamiltonian systems. Preprint 97-142, mp_arc@math.utexas.edu, to appear in J. Differential Equations, 1999.

12. D.V. Treschev. Hyperbolic tori and asymptotic surfaces in Hamiltonian systems. Russian J. Math. Phys., 2(1):93-110, 1994.

13. P.J. Holmes and J.E. Marsden. Melnikov's method and Arnold diffusion for perturbations of integrable Hamiltonian systems. J. Math. Phys., 23:669-675, 1982.

14. S. Wiggins. Global bifurcations and chaos: analytical methods, volume 73 of Appl. Math. Sci. Springer, New York, 1988.

15. C. Robinson. Horseshoes for autonomous Hamiltonian systems using the Melnikov integral. Ergodic Theory Dynam. Systems, 8:395-409, 1988.

16. A. Delshams and R. Ramírez-Ros. Melnikov potential for exact symplectic maps. Comm. Math. Phys., 190:213-245, 1997.

17. C. Simó. Averaging under fast quasiperiodic forcing. In J. Seimenis, editor, Hamiltonian Mechanics: Integrability and Chaotic Behavior, volume 331 of NATO ASI Ser. B: Phys., pages 13-34. Held in Torun, Poland, 28 June-2 July 1993. Plenum, New York, 1994.

18. A. Delshams, V. Gelfreich, A. Jorba, and T.M. Seara. Splitting of separatrices for (fast) quasiperiodic forcing. In C. Simó, editor, Hamiltonian systems with three or more degrees of freedom, volume 533 of NATO ASI Ser. C: Math. Phys. Sci., pages 367-371. Kluwer Acad. Publ., Dordrecht, Holland, 1999.

19. G. Gallavotti. Twistless KAM tori, quasi flat homoclinic intersections, and other cancellations in the perturbation series of certain completely integrable Hamiltonian systems. A review. Rev. Math. Phys., 6(3):343411, 1994.

20. A. Delshams and T.M. Seara. Splitting of separatrices in Hamiltonian systems with one and a half degrees of freedom. Math. Phys. Electron. J., $3(4): 1-40,1997$.

21. S.-N. Chow and J.K. Hale. Methods of bifurcation theory, volume 251 of Grundlehren Math. Wiss. Springer-Verlag, New York, 1982.

Internet access: The authors' quoted preprints are available in the Preprints pages of http://www-ma1.upc.es, or in the pub/preprints directory of ftp://ftp-ma1.upc.es.

E-mails of the authors: amadeu@ma1.upc.es, gutierrez@ma2.upc.es. 